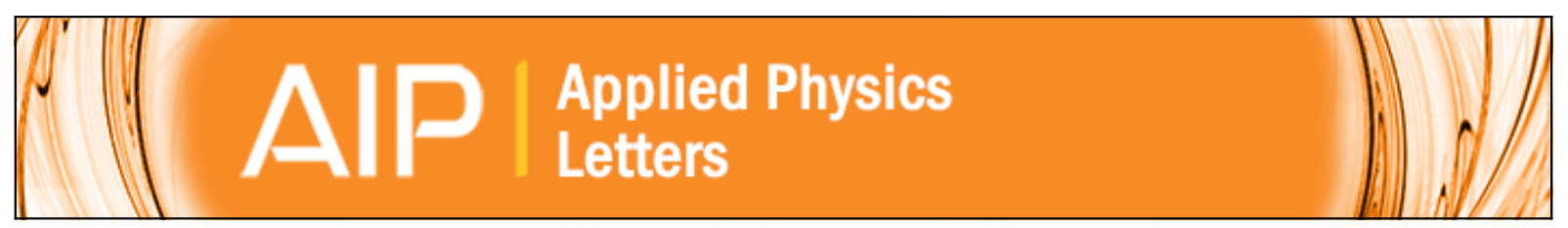

\title{
Scalable self-assembled reduced graphene oxide transistors on flexible substrate
}

Zhenxing Wang, Siegfried Eigler, and Marcus Halik

Citation: Applied Physics Letters 104, 243502 (2014); doi: 10.1063/1.4884064

View online: http://dx.doi.org/10.1063/1.4884064

View Table of Contents: http://scitation.aip.org/content/aip/journal/apl/104/24?ver=pdfcov

Published by the AIP Publishing

\section{Articles you may be interested in}

Manipulation of graphene work function using a self-assembled monolayer

J. Appl. Phys. 116, 084312 (2014); 10.1063/1.4894392

Poly (methyl methacrylate) as a self-assembled gate dielectric for graphene field-effect transistors

Appl. Phys. Lett. 104, 083106 (2014); 10.1063/1.4866338

Phosphonic acid self-assembled monolayer and amorphous hafnium oxide hybrid dielectric for high performance polymer thin film transistors on plastic substrates

Appl. Phys. Lett. 95, 113305 (2009); 10.1063/1.3231445

Flexible organic field-effect transistors fabricated by the electrode-peeling transfer with an assist of selfassembled monolayer

Appl. Phys. Lett. 82, 4373 (2003); 10.1063/1.1583867

Nanoscale organic transistors based on self-assembled monolayers

Appl. Phys. Lett. 80, 847 (2002); 10.1063/1.1445804

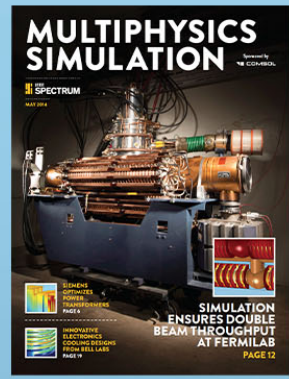

Free online magazine

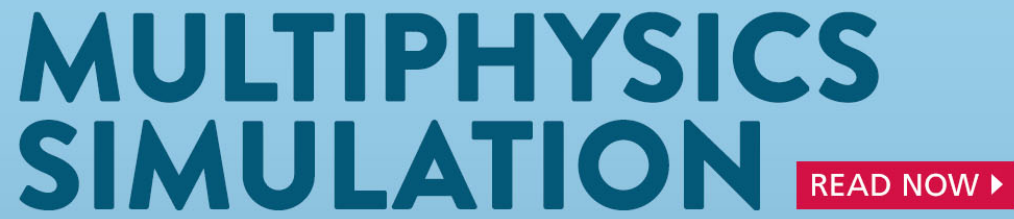




\title{
Scalable self-assembled reduced graphene oxide transistors on flexible substrate
}

\author{
Zhenxing Wang, ${ }^{1}$ Siegfried Eigler, ${ }^{2,3}$ and Marcus Halik ${ }^{1, a)}$ \\ ${ }^{1}$ Organic Materials \& Devices (OMD), Institute of Polymer Materials, Friedrich-Alexander-University \\ Erlangen-Nürnberg (FAU), Martensstraße 7, 91058 Erlangen, Germany \\ ${ }^{2}$ Department of Chemistry and Pharmacy, Friedrich-Alexander-University Erlangen-Nürnberg (FAU), \\ Henkestraße 42, 91054 Erlangen, Germany \\ ${ }^{3}$ Institute of Advanced Materials and Processes (ZMP), Friedrich-Alexander-University Erlangen-Nürnberg \\ (FAU), Dr.-Mack-Straße 81, 90762 Fürth, Germany
}

(Received 15 April 2014; accepted 5 June 2014; published online 17 June 2014)

\begin{abstract}
To enable graphene oxide (GO) flakes for application based on solution processable technology, we show that they can be self-assembled from solution on flexible substrate driven by a Coulomb interaction with the self-assembled monolayer (SAM). Field-effect transistors exhibit a high hole mobility around $14 \mathrm{~cm}^{2} / \mathrm{V} \cdot \mathrm{s}$ after a reduction process from $\mathrm{GO}$ to reduced $\mathrm{GO}(\mathrm{rGO})$, and meanwhile the device resistance shows a linear scaling behavior with the channel length. Due to the flexibility of the SAM, the device parameters maintain stable, while different strains are applied to the substrate. This approach makes the combination of rGO and SAM suitable for low-cost flexible applications. (C) 2014 AIP Publishing LLC. [http://dx.doi.org/10.1063/1.4884064]
\end{abstract}

Graphene is well developed for high-performance electronics, such as transistors with high-frequency response and ambipolar frequency operation. ${ }^{1-3}$ Besides the applications on rigid substrates, the compatibility of graphene with flexible substrates also makes it a promising candidate. ${ }^{4}$ Due to the excellent mechanical properties, they are suitable for developing flexible devices with considerably high performance. A transfer technique is required to place graphene on flexible substrates when chemical vapor deposition (CVD) or epitaxial method is involved in the synthetic process of graphene. ${ }^{5,6}$ However, solution processable graphene material, such as functionalized graphene or graphene oxide (GO), is more favorable for the device fabrication on flexible substrates. ${ }^{4,7}$ Due to the possibility of realizing self-assembly of active materials, the whole process becomes simpler and more cost-efficient. Besides field-effect transistors (FETs), functional memories and conductive electrodes have been also realized based on $\mathrm{GO}$ or reduced $\mathrm{GO}(\mathrm{rGO})$ for flexible applications. $^{7,9-13}$ As gate dielectric, self-assembled monolayers (SAMs) were reported to be suitable for low voltage operation of graphene based transistors. Additionally, functional SAM molecules can drive the self-assembly process of graphene-based materials. ${ }^{14,15}$ By using different SAM molecules, the driving forces for functionalized graphene deposition can be tuned as well as the electrical properties of devices. ${ }^{15}$ However, Coulomb interaction is proved to yield the best formation of the active thin films. ${ }^{14}$

In this work, we show that the self-assembly of GO on SAM can be carried out on a flexible substrate, and scalable functional FET devices can be prepared after the removal of oxygen functional groups of GO. Due to the extreme flexibility of the SAM with a considerably long alkyl chain, the whole system is not sensitive to the strain applied to the substrate. The scalability with channel length maintains for

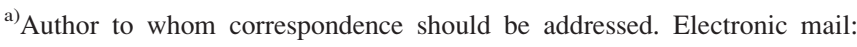
marcus.halik@fau.de
}

devices with compressive strain, tensile strain, or without any strain. This work provides a solution to stabilize the graphene FET on a flexible substrate under different strain levels, which is critical for the large scale production of flexible low-cost solution processable graphene-based electronics.

Polyethylene naphthalate (PEN) plastic substrate with a thickness of $125 \mu \mathrm{m}$ (DuPont Teijin Films, Wilton, UK) is used after a pre-shrinkage treatment. The thermal treatment keeps the substrate at $150^{\circ} \mathrm{C}$ for $5 \mathrm{~h}$ and followed by natural cooling for overnight (Memmert UN 110 universal oven). During the whole treatment, the substrate is fixed between two glass wafers to prevent it from curving. The device fabrication follows a similar photolithographic procedure (based on Karl Suss MA6 mask aligner) as described in the previous work for rigid substrates. ${ }^{14}$ The architecture of the device is shown in Figure 1(a), with gate electrode of $30 \mathrm{~nm}$ thick thermal evaporated aluminum, source and drain electrodes of $30 \mathrm{~nm}$ thick thermal evaporated gold (by evaporator UNIVEX 300). The SAM molecule 12-mercaptododecylphosphonic acid (HS$\mathrm{C}_{12}$-PA) is used to provide an adhesion layer for the source/ drain gold electrodes, and the molecule 3-methyl-1-(12-phosphonododecyl)imidazolium bromide (IMI- $\mathrm{C}_{12}-\mathrm{PA}$ ) provide the driving force, i.e., Coulomb attraction, ${ }^{14}$ during the process of self-assembly of GO. GO is prepared according to our recently published procedure. ${ }^{16}$ Typically, graphite is oxidized in sulfuric acid and potassium permanganate is used as oxidant together with sodium nitrate. The oxidation temperature is between 5 and $10^{\circ} \mathrm{C}$ and the temperature is maintained during work-up and purification of the reaction mixture. After $16 \mathrm{~h}$ of oxidation time, the reaction mixture is slowly diluted with water, avoiding heating of the reaction mixture. Manganese salts are solubilized using diluted hydrogen peroxide. Afterwards, graphite oxide is purified by centrifugation and re-dispersion in water until the $\mathrm{pH}$ of the supernatant is neutral. Delamination of graphite oxide to GO is achieved by using a bath sonication. This low temperature is crucial to reduce the defect density of resulted GO. ${ }^{17}$ Furthermore, the functional 
(a)

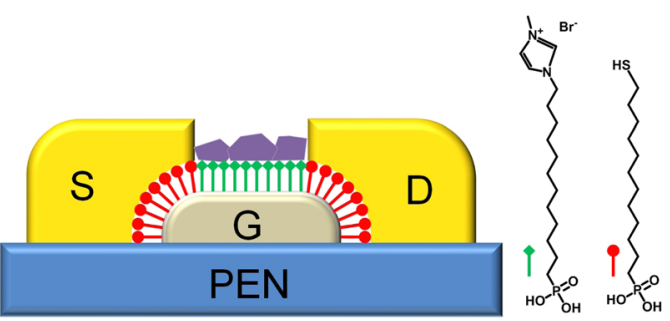

(b)

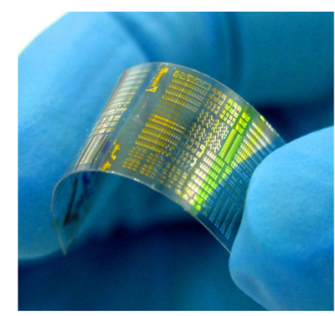

(c)

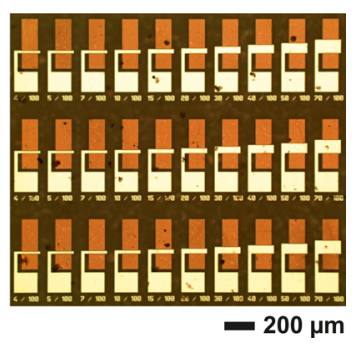

FIG. 1. (a) Geometry of the device with source (S), drain (D), and gate (G) electrodes, as well as the SAM molecule structure of IMI-C $12-\mathrm{PA}$ and HS- $\mathrm{C}_{12}$-PA. (b) The plastic chip with bending applied. (c) The optical microscope image showing the devices we measured. They have varied channel length ranging from $4 \mu \mathrm{m}$ to $70 \mu \mathrm{m}$ from left to right and the channel width is $100 \mu \mathrm{m}$ for all devices.

groups of this type of GO are mainly hydroxyl groups, epoxy groups, and organosulfate groups on both sides of the basal plane. ${ }^{18}$ Especially, organosulfate is responsible for electrostatic interactions with the IMI- $\mathrm{C}_{12}$-PA functionalized surface. Chemical reduction of GO with a mixture of hydroiodic acid (HI) and trifluoroacetic acid (TFA) yields graphene with an average defect density of $0.3 \%$ (as determined by scanning Raman microscopy). ${ }^{17,19}$ The analysis of GO reveals flakes of graphene of mono-layer in average and the mean flake size is about $5 \mu \mathrm{m} .{ }^{19}$ This type of functionalized graphene can be symbolized according to the recent systematic study as ${ }^{5 \mu \mathrm{m}, 0.3 \%} \mathrm{G}_{1}-\left[(\mathrm{OH})_{\mathrm{x}}(\mathrm{O})_{\mathrm{y}}\left(\mathrm{OSO}_{3} \mathrm{H}\right)_{\mathrm{z}}\right]_{50 \%} /\left(\mathrm{H}_{2} \mathrm{O}\right)_{8 \%}{ }^{20,21}$ For clearance, the abbreviation $\mathrm{GO}$ is used for this functionalized graphene throughout the manuscript. The samples are simply dipped in the GO dispersion $(\sim 0.1 \mathrm{mg} / \mathrm{ml})$ for $24 \mathrm{~h}$ with a temperature of about $7^{\circ} \mathrm{C}$ to ensure that organosulfate is not hydrolyzed and remains stable bound to enable electrostatic deposition $^{22}$ and afterwards washed with distilled water and dried with nitrogen blow. The following reduction is carried out in the vapor of HI and TFA for $2 \mathrm{~min}$. The plastic substrate with functional devices is shown in Figure 1(b). The rGO flakes are characterized with Raman spectroscopy performed on Horiba Jobin Yvon LabRAM Aramis confocal Raman spectrometer at laser excitation wavelength of $532 \mathrm{~nm}$ and energy of $0.2 \mathrm{~mW}$. The electric properties of devices are measured in ambient with a DC probe station connected to Agilent B1500A semiconductor analyzer.

The devices series consist of transistors with different channel length ranging from $4 \mu \mathrm{m}$ to $70 \mu \mathrm{m}$ and an identical channel width of $100 \mu \mathrm{m}$, with the optical microscope image shown in Figure 1(c). In Figure 2(a), scanning Raman microscopy was used to image the channel region of a FET device by using a step-size increment of $2 \mu \mathrm{m}$. The G-band intensity can be used to visualize the number of layer of rGO in the channel region. ${ }^{19}$ The overall quality of rGO was checked before by the scanning Raman spectroscopic analysis of a rGO film on $\mathrm{Si} / \mathrm{SiO}_{2}$ substrate, and the average defect density of $0.3 \%$ is confirmed to be yielded by the reduction of GO with HI and TFA. On PEN substrate, Raman spectroscopy is partially hampered due to thermal instability of the substrate (laser power of $0.2 \mathrm{~mW}$ ) and Raman signals of the PEN substrate as shown in Figure 2(b). Therefore, the defect density cannot be directly determined on the PEN substrate. However, we assigned the $I_{\mathrm{G}}$ intensity to monolayer graphene and bilayer graphene (dark blue), few layer graphene (light blue), and multi-layer graphene (yellow) in Figure 2(c). The substrate region is marked in black. From these measurements, we estimate the coverage of channel region to about 50\% and Figure 2(a) indicates that conductive paths are available within the channel region of the FET device.

We bend the substrate by mounting the samples on metal rods with fixed curvature to apply compressive or tensile strain on the device. The strain $S$ can be estimated with $D / 2 R$, where $D$ is the thickness of the substrate and $R$ is the radius for the bending. ${ }^{23}$ For the bending radius of $2 \mathrm{~cm}$, the corresponding strain is about $0.6 \%$. The electric properties are measured under different strain conditions, with $0.6 \%$ (a)

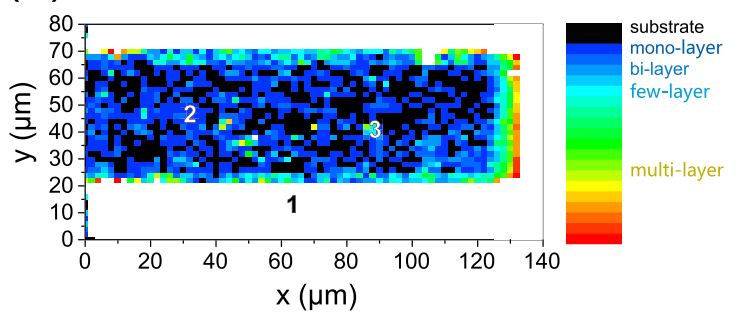

(c)

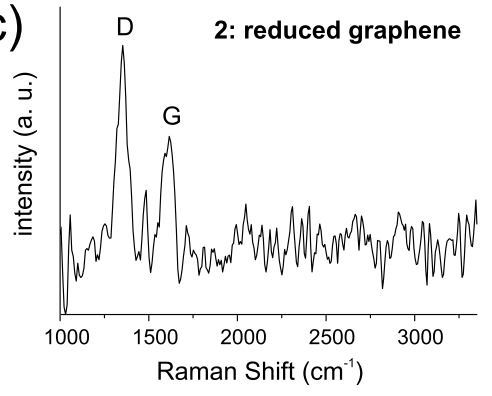

(b)

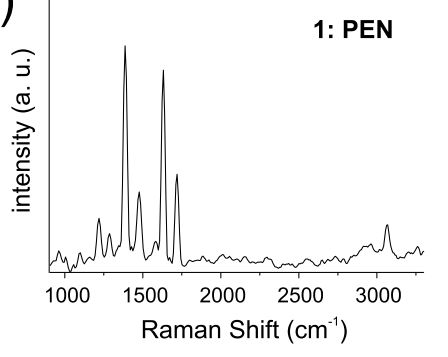

(d)

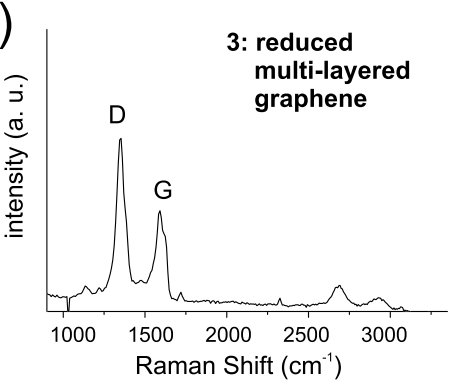

FIG. 2. Raman spectrum characteristic of the device. (a) Scanning Raman microscopic image of the channel region of rGO mapping the intensity of the G-peak $\left(I_{\mathrm{G}}\right)$, which is correlated to the number of layers. (b) Raman spectrum of PEN substrate from location 1. (c) Raman spectrum of a flake (location 2) of reduced GO on PEN substrate. (d) Raman spectrum of a multi-layer region (location 3) of reduced GO on PEN substrate. 


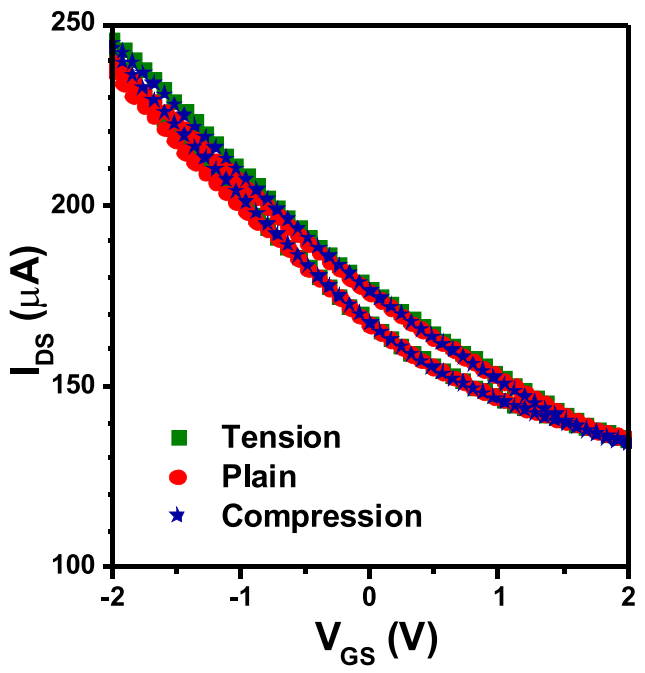

FIG. 3. The transfer curves for the same device under different strain: tensile strain, compressive strain, and plain without strains. The channel length is $40 \mu \mathrm{m}$ and the channel width is $100 \mu \mathrm{m}$. The drain voltage $V_{\mathrm{ds}}$ applied is $1 \mathrm{~V}$. The measurements are running with sweeping gate voltage from $-2 \mathrm{~V}$ to $2 \mathrm{~V}$ (set of curves with lower current), and back from $2 \mathrm{~V}$ to $-2 \mathrm{~V}$ (set of curves with higher current).

compressive strain, with $0.6 \%$ tensile strain, and without any strain. The corresponding transfer curves for a device with $40 \mu \mathrm{m}$ channel length are shown in Figure 3. These curves are highly identical with each other, especially the behavior around the Dirac point, indicating that the rGO FET devices can work rather stable at different bending status. In a previous work, rGO FETs are used as strain sensors, and it was reported in contrast that rGO flakes are ultrasensitive to the applied strain. ${ }^{24}$ Comparing the dielectric of poly-4-vinyl phenol (PVP) in the previous report, the SAM with long alkyl chain $\left(\mathrm{C}_{12}\right)$ here in our work provides much more flexibility to the supported rGO, and thus makes the device very stable while different strain is applied. Besides this, we are able to carry out low voltage operation down to $V_{\mathrm{GS}}=2 \mathrm{~V}$ based on the ultrathin hybrid dielectric of aluminum oxide and SAM, which is also critical for circuitry design and heat
TABLE I. Parameters for the flexible device. Transconductance and hole mobility are extracted while $V_{\mathrm{ds}}=1 \mathrm{~V}$ and $V_{\mathrm{gs}}=-1.44 \mathrm{~V}$.

\begin{tabular}{|c|c|c|c|c|}
\hline & $\begin{array}{c}\text { Transconductance } \\
(\mu \mathrm{S})\end{array}$ & $\begin{array}{l}\text { Hole mobility } \\
\left(\mathrm{cm}^{2} / \mathrm{V} \cdot \mathrm{s}\right)\end{array}$ & $\begin{array}{c}\text { Contact } \\
\text { resistance } \\
(\mathrm{k} \Omega \cdot \mu \mathrm{m})\end{array}$ & $\begin{array}{c}\text { Sheet } \\
\text { resistance }(\mathrm{k} \Omega)\end{array}$ \\
\hline Tension & 37.5 & 14.8 & 245 & 10.4 \\
\hline Plain & 35.6 & 14.0 & 249 & 10.4 \\
\hline Compression & 37.2 & 14.7 & 252 & 10.4 \\
\hline
\end{tabular}

dissipation. $^{25,26}$ The transconductance for the same device under different strains is shown in Table I. Based on the measured average capacitance of $1.02 \mu \mathrm{F} / \mathrm{cm}^{2}$, the hole mobility for linear region is also calculated and listed in Table I, by utilizing a transport model for thin-film transistors (TFTs). ${ }^{27}$ The mobility of holes in the device is around $14 \mathrm{~cm}^{2} / \mathrm{V} \cdot \mathrm{s}$, which is rather high among the results with solution processable $\mathrm{rGO}^{24,28}$ and meanwhile quite stable no matter what kind of strain is applied. We attribute this high mobility to the low defect density of our rGO flakes.

We measured the transfer curves of devices with varied channel lengths, and the minimum resistance for different channel lengths was extracted. The scaling properties of device resistance with channel width are shown in Figure 4, with different strain applied. The total resistance of a rGO FET $\left(R_{\text {total }}\right)$ can be simply divided into three parts, which are the contact resistance $\left(R_{\text {cont }}\right)$, the resistance between different rGO flakes $\left(R_{\text {inter }}\right)$, and the resistance of rGO layer $\left(R_{\text {rgo }}\right) .{ }^{29}$ All the three parts are inversely proportional to the channel width $W_{\mathrm{C}}$, and only $R_{\text {inter }}$ and $R_{\text {rgo }}$ are proportional to the channel length $L_{\mathrm{C}}$

$$
R_{\text {total }}=R_{\text {cont }}\left(\frac{1}{W_{\mathrm{C}}}\right)+R_{\text {inter }}\left(\frac{L_{\mathrm{C}}}{W_{\mathrm{C}}}\right)+R_{\mathrm{rgo}}\left(\frac{L_{\mathrm{C}}}{W_{\mathrm{C}}}\right) .
$$

Therefore, the relation between device resistance and channel length can be fitted to a linear relation. From the linear fitting as shown in Figure 4, one can roughly estimate the contact resistance $R_{\text {cont }}$ directly from the y-intercept of the (a)

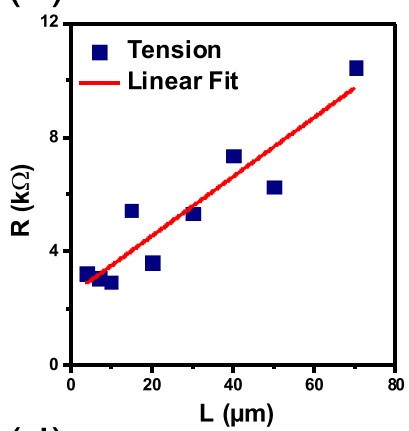

(d)

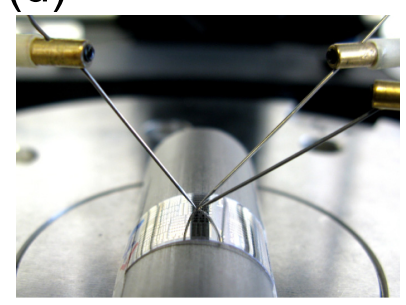

(b)

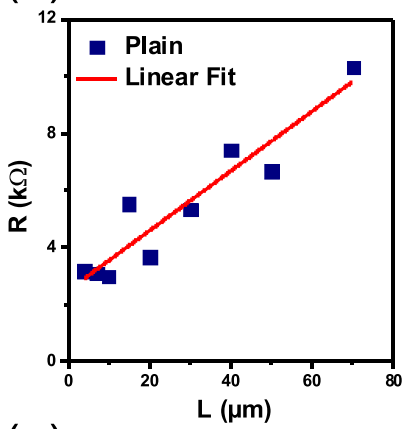

(e)

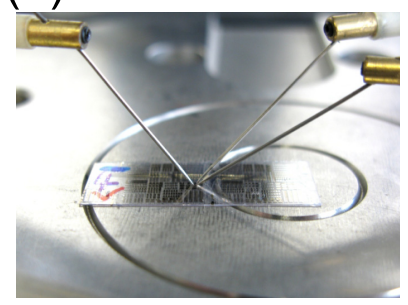

(c)

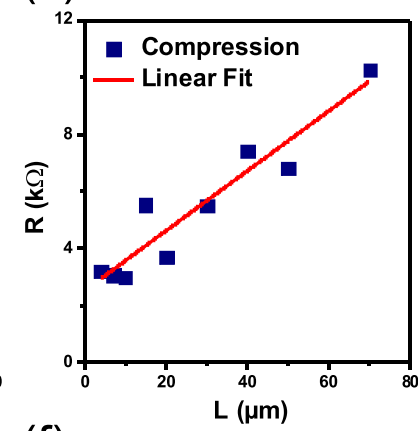

(f)

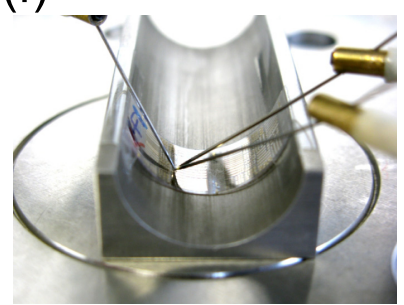

FIG. 4. The device scaling property of the same devices applied (a) with tensile strain, (b) without strain, (c) with compressive strain. The channel width of all the devices is $100 \mu \mathrm{m}$, and the applied drain voltage is $1 \mathrm{~V}$. (d)-(f) show the measurement setups for (a)-(c), respectively. 
line. The calculated normalized resistance $R_{\text {cont }} W_{\mathrm{C}}$ is shown in Table I as well. Besides, one can get the slope of the fitting line as $104 \Omega / \mu \mathrm{m}$ for all strain conditions. Based on the channel width of $100 \mu \mathrm{m}$, the sheet resistance $R_{\square}$ of the channel can be calculated as $10.4 \mathrm{k} \Omega$. As one can see from Table I, all the important parameters under different strain conditions are similar to each other, strongly proving that our system is insensitive to the bending and suitable for applications based on flexible substrate, which can be attributed to the utilization of SAM.

In conclusion, the GO flakes can be well self-assembled on flexible substrate with SAM on the surface. Due to the flexibility of the SAM, the FET behaves stably no matter what kind of strain is applied or without any strain. Besides the transfer curves, the device resistance scaling property is also quite stable. This work provides an approach that could stabilize the performance of rGO based FETs, making this kind of application possible.

We gratefully acknowledge the German Research Council (DFG) with the Collaborative Research Center (SFB-953), Grant No. EI 938/3-1, and the Erlangen Graduate School of Molecular Science (GSMS) for financial support. We appreciate Professor Andreas Hirsch for providing the scientific research environment.

${ }^{1}$ F. Schwierz, Nat. Nanotechnol. 5, 487 (2010).

${ }^{2}$ Z. Wang, Z. Zhang, and L.-M. Peng, Chin. Sci. Bull. 57, 2956 (2012).

${ }^{3}$ N. O. Weiss, H. Zhou, L. Liao, Y. Liu, S. Jiang, Y. Huang, and X. Duan, Adv. Mater. 24, 5782 (2012).

${ }^{4}$ B. K. Sharma and J.-H. Ahn, Solid State Electron. 89, 177 (2013).

${ }^{5}$ S. M. Kim, E. B. Song, S. Lee, J. Zhu, D. H. Seo, M. Mecklenburg, S. Seo, and K. L. Wang, ACS Nano 6, 7879 (2012).

${ }^{6}$ L. Gao, W. Ren, H. Xu, L. Jin, Z. Wang, T. Ma, L.-P. Ma, Z. Zhang, Q. Fu, L.-M. Peng, X. Bao, and H.-M. Cheng, Nat. Commun. 3, 699 (2012).

${ }^{7}$ Q. He, S. Wu, S. Gao, X. Cao, Z. Yin, H. Li, P. Chen, and H. Zhang, ACS Nano 5, 5038 (2011).
${ }^{8}$ D.-M. Sun, C. Liu, W.-C. Ren, and H.-M. Cheng, Small 9, 1188 (2013).

${ }^{9}$ A. Rani, J. Song, M. Lee, and J. Lee, Appl. Phys. Lett. 101, 233308 (2012).

${ }^{10}$ J. Liu, Z. Yin, X. Cao, F. Zhao, L. Wang, W. Huang, and H. Zhang, Adv. Mater. 25, 233 (2013).

${ }^{11}$ G. Eda, G. Fanchini, and M. Chhowalla, Nat. Nanotechnol. 3, 270 (2008).

${ }^{12}$ J. Wang, M. Liang, Y. Fang, T. Qiu, J. Zhang, and L. Zhi, Adv. Mater. 24, 2874 (2012).

${ }^{13}$ H. Tian, Y. Yang, D. Xie, T.-L. Ren, Y. Shu, C.-J. Zhou, H. Sun, X. Liu, and C.-H. Zhang, Nanoscale 5, 890 (2013).

${ }^{14}$ Z. Wang, S. Mohammadzadeh, T. Schmaltz, J. Kirschner, A. Khassanov, S. Eigler, U. Mundloch, C. Backes, H.-G. Steinrück, A. Magerl, F. Hauke, A. Hirsch, and M. Halik, ACS Nano 7, 11427 (2013).

${ }^{15}$ N. Cernetic, S. Wu, J. A. Davies, B. W. Krueger, D. O. Hutchins, X. Xu, H. Ma, and A. K.-Y. Jen, Adv. Funct. Mater. 24, 3464 (2014).

${ }^{16}$ S. Eigler, M. Enzelberger-Heim, S. Grimm, P. Hofmann, W. Kroener, A. Geworski, C. Dotzer, M. Röckert, J. Xiao, C. Papp, O. Lytken, H.-P. Steinrück, P. Müller, and A. Hirsch, Adv. Mater. 25, 3583 (2013).

${ }^{17}$ S. Eigler, S. Grimm, M. Enzelberger-Heim, P. Müller, and A. Hirsch, Chem. Commun. 49, 7391 (2013).

${ }^{18}$ S. Eigler, C. Dotzer, F. Hof, W. Bauer, and A. Hirsch, Chem. Eur. J. 19, 9490 (2013).

${ }^{19}$ S. Eigler, F. Hof, M. Enzelberger-Heim, S. Grimm, P. Müller, and A. Hirsch, J. Phys. Chem. C 118, 7698 (2014).

${ }^{20}$ S. Eigler and A. Hirsch, "Chemie mit Graphen und Graphenoxid - Eine Herausforderung für Synthesechemiker," Angew Chem. (to be published).

${ }^{21}$ S. Eigler and A. Hirsch, "Chemistry with Graphene and Graphene Oxide Challenges for Synthetic Chemists,” Angew Chem. Int. Ed. (to be published).

${ }^{22}$ S. Eigler, S. Grimm, F. Hof, and A. Hirsch, J. Mater. Chem. A 1, 11559 (2013).

${ }^{23}$ S.-T. Han, Y. Zhou, and V. A. L. Roy, Adv. Mater. 25, 5425 (2013).

${ }^{24}$ T. Q. Trung, N. T. Tien, D. Kim, M. Jang, O. J. Yoon, and N.-E. Lee, Adv. Funct. Mater. 24, 117 (2014).

${ }^{25}$ M. Halik, H. Klauk, U. Zschieschang, G. Schmid, C. Dehm, M. Schutz, S. Maisch, F. Effenberger, M. Brunnbauer, and F. Stellacci, Nature 431, 963 (2004).

${ }^{26}$ H. Klauk, U. Zschieschang, J. Pflaum, and M. Halik, Nature 445, 745 (2007).

${ }^{27}$ M. C. Hamilton, S. Martin, and J. Kanicki, Chem. Mater. 16, 4699 (2004).

${ }^{28}$ T. Q. Trung, N. T. Tien, D. Kim, J. H. Jung, O. J. Yoon, and N.-E. Lee, Adv. Mater. 24, 5254 (2012).

${ }^{29}$ T. Kobayashi, N. Kimura, J. Chi, S. Hirata, and D. Hobara, Small 6, 1210 (2010). 